\title{
La metodología de resolución de problemas como investigación (MRPI): una propuesta indagativa para desarrollar la competencia científica en alumnos que cursan un progra- ma de diversificación
}

\section{The methodology of problem-solving as an investigation (MPSI): an inquiry approach for developing the scientific competence in diversification program students}

\author{
Fernando Pavón Martínez \\ fpavon@ucm.es \\ M.a Mercedes Martínez Aznar \\ Departamento de Didáctica de las Ciencias Experimentales, Facultad de Educación-Centro de Formación del Profesorado \\ Universidad Complutense, Madrid \\ mtzaznar@ucm.es
}

RESUMEN • Para la enseñanza-aprendizaje de las ciencias, las organizaciones internacionales recomiendan el uso de metodologías basadas en la indagación en lugar de los métodos tradicionales. Estas diferentes metodologías son clave en los programas de diversificación. Con el presente estudio se pretende valorar si un método indagativo como es la metodología de resolución de problemas como investigación (MRPI) es adecuado para desarrollar la competencia científica en el ámbito científicotecnológico de cuarto de ESO. Por ello, se ha estudiado la evolución de los alumnos en la resolución de situaciones problemáticas para diferentes dimensiones de la competencia científica. Los resultados indican que la MRPI ayuda a desarrollar estas diferentes dimensiones competenciales con un buen nivel de satisfacción por parte del alumno.

PALABRAS CLAVE: programa de diversificación; ámbito científico-tecnológico; competencia científica, indagación; metodología de resolución de problemas como investigación (MRPI).

ABSTRACT - International organizations recommend the use of inquiry-based methods for teachinglearning of sciences, instead of the traditional teaching deductive methods. These different methodologies are key in diversification programs. This study aims to assess whether an inquiry method such as the Methodology of Problem-Solving as an Investigation (MPSI) is suitable to develop the scientific competence in scientific-technological area in the 4th year of ESO. Therefore, we have studied the evolution of the students in the resolution of problematic situations for different dimensions of scientific competence. The results indicate that the MPSI helps to develop these different dimensions of competence with a good level of satisfaction on the part of the student.

KEYWORDS: diversification program; scientific-technological area; scientific competence; Inquirybased Science Education (IBSE); methodology of problem-solving as an investigation (MPSI). 


\section{INTRODUCCIÓN}

El presente estudio se centra en la enseñanza de las ciencias dentro del ámbito científico-tecnológico (ACT) de un programa de diversificación curricular. Estos programas se caracterizan por contar con un alumnado que presenta dificultades de aprendizaje que le impiden alcanzar los niveles competenciales que establece el currículo ordinario de la ESO. Es, por tanto, en estos programas donde se hace patente, más aún si cabe, la necesidad de un cambio metodológico. La propia LOE especifica en su art. 27 que «en la definición de las enseñanzas mínimas de la etapa [ESO] se incluirán las condiciones básicas para establecer las diversificaciones del currículo [...] en este supuesto, los objetivos de la etapa se alcanzarán con una metodología específica a través de una organización de contenidos, actividades prácticas y, en su caso, de materias, diferente a la establecida con carácter general» (MEC, 2006: 17171).

Todo parece indicar que, primordialmente, en un programa de diversificación, se hace necesario seguir las recomendaciones generales para enseñar ciencias de una manera indagativa (Informe Rocard, 2007), puesto que «las propias características del alumnado que cursa este programa aconsejan que el aprendizaje sea lo más funcional posible» (CM, 2007: 8), de tal modo que se produzcan aprendizajes más significativos (Bransford, Brown y Cocking, 2000) y se motive en mayor grado tanto a alumnos como a profesores. De entre todas las opciones, la propuesta que queremos defender aquí es la metodología de resolución de problemas como investigación (MRPI), un método indagativo (Inquiry-Based Science Education, IBSE) ampliamente trabajado en España, en las universidades de Valencia y la Complutense de Madrid, que deseamos extender a los estudiantes de diversificación.

\section{MARCO TEÓRICO}

Desde finales del siglo pasado y principios de este, los currículos oficiales que materializan las reformas educativas han incluido, aunque con diferencias terminológicas y más o menos alcance, la indagación (IBSE) como método de enseñanza y estilo de aprendizaje para desarrollar los contenidos y la competencia científica con la finalidad de alcanzar la educación científica de los escolares y su formación como futuros ciudadanos (Keys y Bryan, 2001; Schwartz, Lederman y Crawford, 2004). Es decir, la indagación es un medio y un fin (Abd-El-Khalick et al., 2004; Prince y Felder, 2006).

En el sistema educativo español la indagación se introduce a través de la competencia científica que los alumnos de ESO habrán de desarrollar y se define como:

... la habilidad progresiva para poner en práctica los procesos y actitudes propios del análisis sistemático y de indagación científica: identificar y plantear problemas relevantes; realizar observaciones [...] con conciencia del marco teórico o interpretativo que las dirige; formular preguntas; localizar, obtener, analizar y representar información cualitativa y cuantitativa; plantear y contrastar soluciones tentativas o hipótesis; realizar predicciones e inferencias de distinto nivel de complejidad; e identificar el conocimiento disponible [...] necesario para responder a las preguntas científicas, y para obtener, interpretar, evaluar y comunicar conclusiones... (MEC, 2007: 687).

Es decir, se hace hincapié en las destrezas propias para hacer indagación y, de forma vaga, se hace mención a algunos aspectos relativos a la naturaleza y epistemología de la indagación científica.

En este sentido, diferentes autores echan en falta que en los documentos curriculares se presenten una definición operativa que permita entender qué es la indagación y su aplicación en las aulas (Anderson, 2002; Abd-El-Khalick et al., 2004). Así, y más allá de la confusión sobre el significado de la indagación, hay otras causas que limitan su acogida entre el profesorado: el creer que está pensada para alumnado de altas capacidades, que requiere mucho tiempo, que es difícil de gestionar, que no se dispone de materiales y recursos adecuados y que hay carencias formativas para su implementación 
(Colburn, 2000; Quintanilla et al., 2010). En consecuencia, la forma más habitual de trabajar las ciencias a nivel escolar está centrada en el profesor, en las explicaciones y presentaciones de los contenidos científicos que luego el alumnado aplica, repetitivamente, en ejercicios y donde «los experimentos se utilizan sobre todo a modo de ilustración» (Informe Rocard, 2007: 10) mediante guiones que determinan su finalidad y el procedimiento de realización. Este tipo de instrucción ha recibido diferentes apelativos pero, de forma genérica, se denomina tradicional, expositiva, deductiva o no-indagativa (Domin, 2007; Informe Rocard, 2007).

Como alternativa a estos enfoques están los denominados no-tradicionales, centrados en el estudiante, inductivos o indagativos, que tienen como eje central «plantear a los alumnos un desafío y que aprendan lo que sea necesario saber para afrontar el reto" (Prince y Felder, 2007: 14). Dentro de estos se encuentran el descubrimiento, la resolución de problemas, el estudio de caso, los proyectos y el just-in-time que se diferencian en el tipo y naturaleza del desafío y en la cantidad y calidad de las orientaciones que reciben por parte del profesor para resolverlo.

Desde el punto de vista del aprendizaje, todos estos métodos tienen en común que los estudiantes, al enfrentarse al reto, ya sea de tipo teórico o práctico, de laboratorio/experimental, construyen conocimiento interactuando con el contexto social y físico y mediante el andamiaje que proporciona el profesor (Tortosa, 2013; Abd-El-Khalick et al., 2004; Hmelo-Silver, 2004). Para determinar la eficacia de los aprendizajes (Hmelo-Silver y Barrows, 2006) se debe considerar el contexto de la tarea y la calidad de su planteamiento, así como su desarrollo, para que promuevan la construcción de conocimientos y de estrategias y destrezas, tanto metodológicas como cognitivas (Bransford, Brown y Cocking, 2000; Hmelo-Silver, Duncan y Chinn, 2007), y fomenten el interés de los escolares hacia la ciencia y su aprendizaje (Hofstein, 2003; Informe Rocard, 2007; Proyecto Primas, 2013).

En este trabajo se asume que la indagación es «el proceso intencional de diagnóstico de problemas, la crítica de los experimentos y distinción de alternativas, la planificación de las investigaciones, la investigación de conjeturas, la búsqueda de información, la construcción de modelos, el debate con los compañeros y la formación de argumentos coherentes» (Linn, Davis y Bell, 2004: 4), definición que caracteriza de forma amplia los componentes de la indagación, incluyendo la naturaleza y las capacidades para hacer indagación y la forma en que los científicos trabajan en el desarrollo del conocimiento científico.

De entre los diferentes métodos indagativos, para este estudio se ha optado por la MRPI (Gil y Martínez-Torregrosa, 1983; Ramírez, Gil y Martínez-Torregrosa, 1994). Esta es una metodología ampliamente trabajada con buenos resultados (Martínez Aznar e Ibáñez, 2006) en diferentes asignaturas como Física (Martínez Aznar y Varela, 1996 y 1997; Varela y Martínez Aznar, 1997), Química (Martínez Aznar y Ovejero, 1997) o Biología (Martínez Aznar e Ibáñez, 2005; Ibáñez y Martínez Aznar, 2005 y 2007), y es perfectamente exportable a diversificación. La MRPI se incluye dentro de los métodos indagativos, es decir, el alumnado trabaja en grupos cooperativos, se enfrenta a situaciones abiertas contextualizadas que debe reformular y definir con precisión, tiene que identificar lo que sabe y lo que necesita conocer, enuncia y contrasta soluciones alternativas y decide cómo proceder para llegar a una posible solución.

En esta ocasión, se traslada la MRPI al área científico-tecnológica de diversificación curricular, sobre la que hay escasa literatura en este sentido (Martínez Aznar y Bárcena, 2013, Boyano, 2000), pues la mayoría de las fuentes documentales se centran en experiencias personales sobre la selección y caracterización del alumnado (Torrego y Leal, 2009), el reducido número de estudiantes por grupo (Verger, 2001), la organización de las aulas (Mohedano, 2000) y el tipo de actividades que se realizan, fundamentalmente ejercicios (Torrego y Leal, 2009).

En la figura 1 se muestra un esquema en el que se aprecia el carácter cíclico de la metodología con sus cinco etapas, y en el anexo 1 se incluye una plantilla que permite su adaptación al ACT y orientar el tipo de andamiaje que debe realizar el profesorado. 


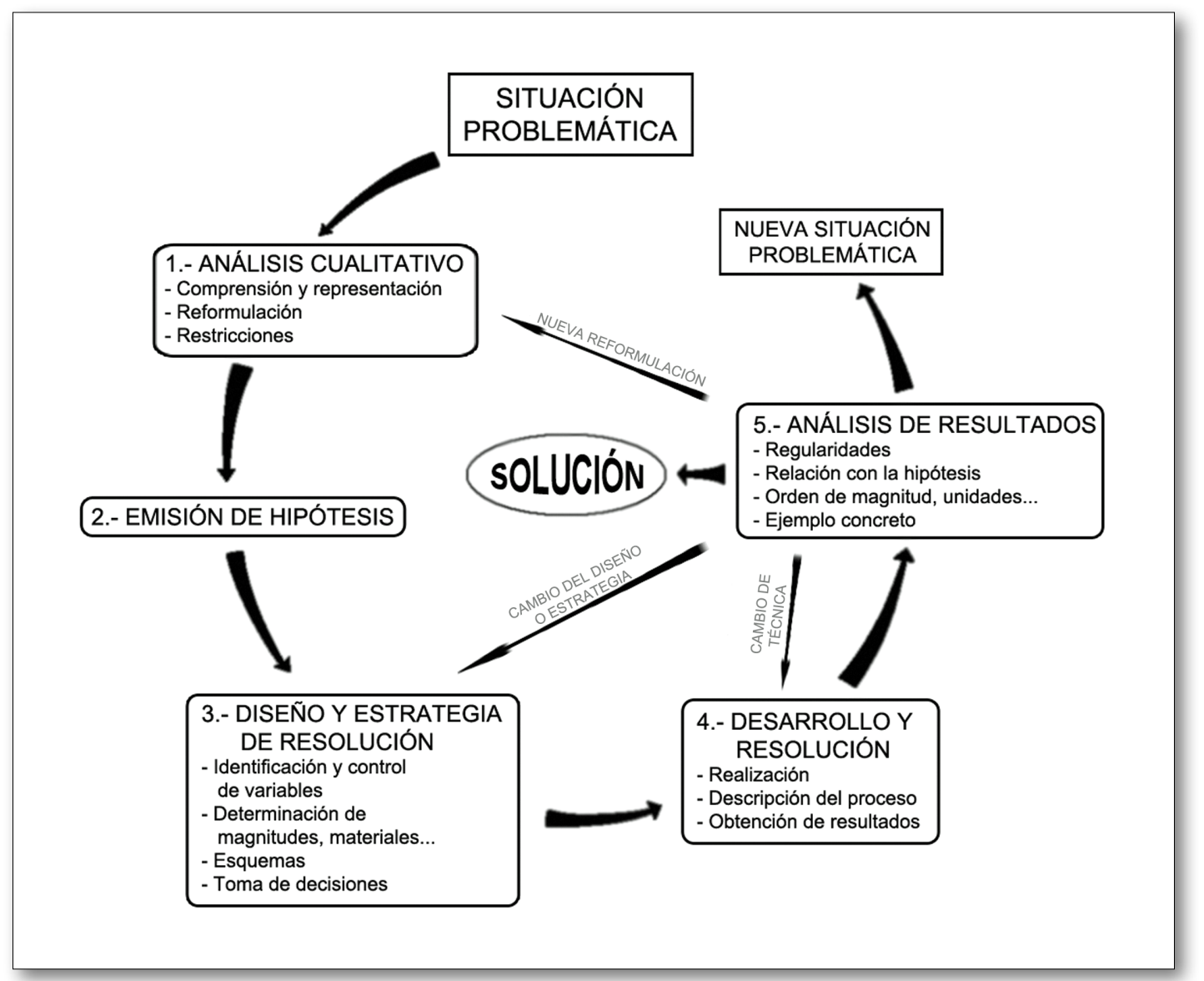

Fig. 1. Esquema general de la metodología de resolución de problemas como investigación (adaptado de Martínez Aznar y Bárcena, 2013).

\section{OBJETIVOS}

El presente trabajo tiene por objetivo general estudiar la eficacia de la MRPI para el desarrollo de la competencia científica en un grupo de alumnos con necesidades especiales de aprendizaje, como puede ser uno de diversificación. Para el contexto del ACT de cuarto de la ESO con el que se ha trabajado y para la unidad didáctica "Cambios físicos y cambios químicos», cabe plantearse las siguientes preguntas:

1. ¿La introducción de la MRPI ayuda a los estudiantes de diversificación a evolucionar en el desarrollo de la competencia científica?

2. ¿El uso de la MRPI permite alcanzar mayores niveles competenciales a alumnos con necesidades especiales de aprendizaje?

3. ¿En qué medida satisface a los alumnos de ACT el uso de un método indagativo como la MRPI?

\section{METODOLOGÍA}

Este trabajo es descriptivo y cualitativo, pues estudia una realidad con la intención de interpretarla según las necesidades y objetivos de investigación planteados (Rodríguez, Gil y García, 1996). Por ello, se han recogido datos escritos de los alumnos al resolver situaciones problemáticas con la MRPI y sus 
respuestas a un cuestionario de satisfacción hacia el método y, aunque no se consideran en este trabajo, se han registrado grabaciones en audio y vídeo con el objeto de poder analizar relaciones más complejas en el aula. Por tanto, dado que «las investigaciones cualitativas [...] están sujetas a las circunstancias de cada ambiente o escenario particular» (Hernández, Fernández y Baptista, 2006: 686), se ha planteado un estudio de caso para analizar la adecuación de la MRPI en un grupo concreto de diversificación.

\section{Muestra de estudio}

Se cuenta con una muestra de carácter incidental constituida por once estudiantes de cuarto de ESO, cinco chicos y seis chicas, entre los 16 y los 18 años, integrados en el ACT del programa de diversificación del Colegio Lourdes de Madrid, con las siguientes características:

- Cinco alumnos son extranjeros, de países tan diversos como India, Ucrania, China o Rumanía; tres presentan dificultades importantes a la hora de expresarse en castellano, tanto hablado como escrito, y dos tienen también una baja autoestima y dificultades para las relaciones sociales.

- Seis alumnos son españoles, de los cuales cuatro presentan un trastorno por déficit de atención e hiperactividad (TDAH). Entre estos últimos, uno requiere medicación, otro presenta un desarrollo intelectual equivalente al de un estudiante de 13 ańos y los otros dos alumnos españoles manifiestan una muy baja autoestima y motivación.

- De los once alumnos, cuatro viven en hogares desestructurados con el padre ausente.

\section{Metodología de aula}

Para el estudio se diseñó una unidad didáctica titulada «Cambios físicos y cambios químicos», basada exclusivamente en la resolución de situaciones problemáticas abiertas (tabla 1) y que permitiera la introducción progresiva de un método indagativo y al mismo tiempo trabajar los contenidos del currículo de diversificación.

En el problema cero, «¿Qué papel absorbe más?», se le plantea al alumno un desafío muy ligado al contexto de la vida cotidiana. Este es un problema ya trabajado con buenos resultados en la bibliografía (por ejemplo, en APU, 1987), que aquí se utilizó como punto de partida previo a la introducción de la MRPI. Así pues, se invitó a los alumnos a que resolvieran de manera individual en sus casas dicho problema, sin especificarles una metodología concreta que seguir. La primera sesión se utilizó para realizar una puesta en común con los alumnos y proponerles una posible solución siguiendo la MRPI a partir de un guion facilitado a estos (anexo I), y así poder comparar las ventajas del uso de la metodología. Las siguientes cinco sesiones se utilizaron para que los alumnos resolvieran el problema 1, «¿Cómo separo una muestra de hierro, cobre, corcho y sal?», que consiste en la reformulación de un problema trabajado a nivel escolar que se propone con el objeto de que los alumnos comiencen a utilizar la MRPI como metodología para resolver problemas. De estas cinco sesiones, las cuatro primeras se invirtieron en que los escolares plantearan su marco teórico y propuesta de resolución, y la última sesión se usó para que pusieran en práctica experimentalmente aquello que habían planificado y extrajeran sus conclusiones. De manera análoga se trabajó el problema 2, «¿Qué hace que un pan sea más esponjoso?», problema basado en una propuesta de la Fundación La Main à la Pâte (2011) para alumnos de educación primaria, que se adaptó convenientemente a los alumnos de diversificación. Se dedicaron cuatro sesiones a que los alumnos plantearan su problema y diseńaran una posible solución y dos sesiones más a que experimentaran su propuesta en el laboratorio y sacaran conclusiones. 
Los escolares desarrollaron todas las fases de la MRPI en grupos cooperativos, en el aula, empleándose, durante cuatro semanas, doce sesiones de cincuenta minutos para la resolución de los tres problemas de la unidad didáctica. Dichos grupos fueron conformados por el tutor del curso, atendiendo a parámetros como la diversidad de género o país de procedencia y las afinidades personales entre los propios individuos (Johnson y Johnson, 2008).

Tabla 1.

Situaciones problemáticas trabajadas

\begin{tabular}{|l|l|l|}
\hline \multicolumn{1}{|c|}{ Problemas } & \multicolumn{1}{c|}{ Contexto } & \multicolumn{1}{c|}{ Forma de abordarlo } \\
\hline 0- ¿Qué papel absorbe más? & Vida cotidiana & Sin método específico \\
\hline 1- ¿Cómo separo una mezcla de hierro, cobre, corcho y sal? & Académico & Con MRPI \\
\hline 2- ¿Qué hace que un pan sea más esponjoso? & Vida cotidiana & Con MRPI \\
\hline
\end{tabular}

\section{Instrumentos y técnicas de recogida de datos y de análisis}

Para tratar de dar respuesta a las preguntas planteadas se utilizaron como instrumentos de recogida de datos, por una parte, las producciones individuales elaboradas por los alumnos sobre las soluciones de las situaciones problemáticas abiertas siguiendo la MRPI, a partir del trabajo en grupos cooperativos, y, por otra, un cuestionario de satisfacción respecto a la MRPI pasado a los alumnos al final del proceso de enseñanza-aprendizaje de la unidad didáctica.

En relación con las producciones escritas y para determinar el nivel de competencia alcanzado por los escolares durante el proceso de enseñanza-aprendizaje, se ha establecido la correspondencia entre las cinco fases de la MRPI y las diferentes dimensiones de la competencia científica propuestas en el currículo oficial que para su análisis se definen de la siguiente manera:

- DC1. Análisis cualitativo de la situación problemática. Vinculada a la capacidad de identificar y plantear problemas en relación con el marco teórico que las rige.

- DC2. Emisión de hipótesis. Relativa a la elección entre explicaciones alternativas y al planteamiento de soluciones tentativas, predicciones y/o inferencias.

- DC3. Diseño de la experimentación y/o estrategia de resolución. Concerniente a la capacidad de canalizar y utilizar los conocimientos construidos en DC1 para diseñar y tomar decisiones sobre qué hacer para resolver el problema.

- DC4. Desarrollo de la experimentación y/o resolución del problema. Relativa a las aptitudes relacionadas con la destreza manual y a la capacidad de observación durante la experimentación.

- DC5. Análisis de los resultados. Referente a la habilidad para dar respuesta a preguntas científicas y para obtener, interpretar, evaluar y comunicar conclusiones.

Para evaluar cada una de estas dimensiones competenciales se han preestablecido unos niveles que faciliten su cuantificación. Dichos niveles se han determinado para cada una de las dimensiones competenciales que se han de valorar, de manera general, tal y como queda reflejado en la tabla 2. 
Tabla 2.

Niveles establecidos para cada dimensión competencial

\begin{tabular}{|c|l|}
\hline Niveles & \multicolumn{1}{|c|}{ Característica de la aportación } \\
\hline Nivel 1 & Irrelevante \\
\hline Nivel 2 & Escasa o con varias carencias importantes \\
\hline Nivel 3 & Satisfactoria pero con alguna carencia relevante \\
\hline Nivel 4 & Muy satisfactoria \\
\hline
\end{tabular}

Así, para la valoración de las producciones de cada estudiante, primero se ha diseñado una solución a las situaciones problemáticas que contemple todos los posibles planteamientos de los alumnos (en el anexo 2 se presenta un ejemplo), y, en segundo lugar, se han establecido los criterios de corrección que definen cada uno de los cuatro niveles para cada situación problemática (en el anexo 3 también se presenta un ejemplo).

Una vez fijados estos niveles para las producciones de los alumnos, y para obtener una visión más global de lo sucedido, se han determinado dos indicadores de logro (ya utilizados en Martínez y Varela, 2009): el indicador de logro 1 (IL1), para poder valorar el éxito relativo alcanzado en cada dimensión competencial, y el indicador de logro 2 (IL2), para identificar el nivel alcanzado por los estudiantes. La tabla 3 resume la característica de cada indicador y su rango.

Tabla 3.

Indicadores de logro considerados en la investigación

\begin{tabular}{|c|l|c|}
\hline Indicadores de logro & \multicolumn{1}{|c|}{ Característica del indicador } & Rango del indicador \\
\hline IL1 & $\begin{array}{l}\text { Diferencia normalizada entre alumnos en los niveles superiores (3-4) } \\
\text { e inferiores (1-2) }\end{array}$ & entre -1 y +1 \\
\hline IL2 & Valor medio alcanzado en cada dimensión competencial & entre 1 y 4 \\
\hline
\end{tabular}

Con esta información se ha procedido a valorar las producciones de los alumnos para poder realizar análisis descriptivos de la muestra estudiada. Así mismo, para analizar la evolución en cada dimensión competencial se han realizado diagramas tipo Bliss, como los ya utilizados en distintas investigaciones didácticas (Ibáñez y Martínez Aznar, 2005; Martínez Aznar y Varela, 2009), que muestran directamente y de manera intuitiva la evolución en el desarrollo de las dimensiones de la competencia científica.

Con respecto al grado de satisfacción de los alumnos en el uso de la MRPI, se ha utilizado el cuestionario extraído de Ibáńez (2003) adaptando el lenguaje a las características particulares del grupo, según se muestra en el anexo 4. El cuestionario, de contestación anónima, consta de doce preguntas o ítems cuyas respuestas están acotadas por una escala de tipo Likert entre 1 y 5 . Con objeto de realizar un análisis más pormenorizado, los valores 1 y 2 se agrupan por un lado, por considerarse respuestas negativas, y los valores 4 y 5 se agrupan por otro, al tratarse de respuestas positivas. El valor intermedio 3 correspondería a los individuos indecisos. Además de la puntuación, cada pregunta se completa con un espacio para responder por qué, lo que permite argumentar la valoración dada. Los doce ítems se pueden agrupar en cuatro categorías, tal y como se muestra en la tabla 4. 
Tabla 4.

Categoría para cada ítem del cuestionario de satisfacción MRPI

\begin{tabular}{|l|l|}
\hline \multicolumn{1}{|c|}{ Categoría } & \multicolumn{1}{c|}{ Ítem } \\
\hline Características de la tarea & $1,2,3,4$ y 5 \\
\hline Autoconfianza & 6,10 y 12 \\
\hline Metodología y papel del profesor & 7 y 11 \\
\hline Transposición a otras materias & 8 y 9 \\
\hline
\end{tabular}

\section{RESULTADOS Y ANÁLISIS}

En este apartado se presentan y analizan los resultados para cada una de las tres preguntas de investigación planteadas en este estudio.

\section{Primera pregunta de investigación}

La primera cuestión planteada era «¿La introducción de la MRPI ayuda a los estudiantes de diversificación a evolucionar en el desarrollo de la competencia científica?».

Para analizar la utilidad de la MRPI en el desarrollo competencial de los escolares se dispone de las tres situaciones problemáticas de la unidad didáctica "Cambios físicos y cambios químicos». A continuación, en la tabla 5 , se muestran los resultados obtenidos por los estudiantes según los procedimientos indicados en el apartado anterior.

Tabla 5.

Resultados obtenidos por los alumnos en los tres problemas abiertos

\begin{tabular}{|c|c|c|c|c|c|c|c|c|c|c|c|c|c|c|c|}
\hline \multirow{3}{*}{$\underset{\stackrel{\Xi}{\Xi}}{\stackrel{\Xi}{\Xi}}$} & \multicolumn{15}{|c|}{ Situaciones problemáticas trabajadas } \\
\hline & \multicolumn{5}{|c|}{ 0. ¿Qué papel absorbe más? } & \multicolumn{5}{|c|}{$\begin{array}{l}\text { 1. ¿Cómo separo una mezcla de } \\
\text { hierro, cobre, corcho y sal? }\end{array}$} & \multicolumn{5}{|c|}{$\begin{array}{l}\text { 2. ¿Qué hace que un pan sea más } \\
\text { esponjoso? }\end{array}$} \\
\hline & DC1* & DC2 & DC3 & DC4 & DC5 & DC1 & DC2 & DC3 & DC4 & DC5 & DC1 & DC2 & DC3 & DC4 & DC5 \\
\hline $1 A^{* *}$ & 2 & 2 & 3 & 1 & 1 & 3 & 3 & 4 & 3 & 1 & 3 & 3 & 3 & 4 & 3 \\
\hline $2 \mathrm{~A}$ & 1 & 2 & 1 & 1 & 1 & 3 & 3 & 4 & 3 & 3 & 3 & 4 & 4 & 3 & 4 \\
\hline $3 \mathrm{~A}$ & 1 & 1 & 2 & 2 & 2 & 3 & 3 & 4 & 3 & 3 & 3 & 4 & 4 & 4 & 4 \\
\hline $4 \mathrm{~A}$ & 1 & 1 & 2 & 3 & 2 & 3 & 3 & 4 & 3 & 3 & 3 & 4 & 4 & 4 & 4 \\
\hline $5 \mathrm{~B}$ & 1 & 1 & 2 & 2 & 2 & 2 & 2 & 3 & 3 & 2 & 2 & 3 & 3 & 4 & 3 \\
\hline $6 \mathrm{~B}$ & 1 & 1 & 2 & 2 & 2 & 2 & 3 & 3 & 3 & 2 & 2 & 3 & 3 & 1 & 2 \\
\hline $7 \mathrm{~B}$ & 1 & 2 & 2 & 3 & 2 & 2 & 3 & 3 & 3 & 3 & 2 & 4 & 4 & 4 & 4 \\
\hline $8 \mathrm{~B}$ & 1 & 4 & 1 & 1 & 1 & 2 & 3 & 3 & 3 & 2 & 2 & 3 & 3 & 4 & 1 \\
\hline $9 \mathrm{C}$ & 1 & 1 & 1 & 1 & 2 & 2 & 2 & 3 & 3 & 2 & 2 & 4 & 4 & 4 & 4 \\
\hline $10 \mathrm{C}$ & 1 & 1 & 3 & 1 & 2 & 2 & 2 & 3 & 3 & 1 & 3 & 4 & 3 & 4 & 4 \\
\hline $11 \mathrm{C}$ & 2 & 2 & 1 & 1 & 2 & 2 & 2 & 3 & 4 & 2 & 2 & 4 & 4 & 4 & 4 \\
\hline
\end{tabular}

* Las distintas dimensiones competenciales se han designado como DC1: Análisis cualitativo de la situación problemática; DC2: Emisión de hipótesis; DC3: Diseño de la experimentación y/o estrategia de resolución; DC4: Desarrollo de la experimentación y/o resolución del problema, y DC5: Análisis de los resultados.

** Para mantener en todo momento el anonimato de los alumnos se les ha asignado un número entre 1 y 11 . La letra que sigue al número asignado a cada alumno (A, B o C) corresponde al grupo de trabajo en el cual el alumno se incluye. Así pues, por ejemplo, el alumno $4 \mathrm{~A}$ es el alumno número 4 que trabaja en el grupo $\mathrm{A}$. 
Para abordar esta primera pregunta de investigación se han comparado los resultados obtenidos en los problemas abiertos 0 y 1 recogidos en la tabla 6 .

Tabla 6.

Frecuencias de los niveles e IL1 para los problemas 0 y 1

\begin{tabular}{|c|c|c|c|c|c|c|c|c|c|c|}
\hline \multirow{4}{*}{ 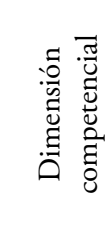 } & \multicolumn{10}{|c|}{ Situaciones problemáticas } \\
\hline & \multicolumn{5}{|c|}{ 0. ¿Qué papel absorbe más? } & \multicolumn{5}{|c|}{$\begin{array}{l}\text { 1. ¿Cómo separo una mezcla de hie- } \\
\text { rro, cobre, corcho y sal? }\end{array}$} \\
\hline & \multicolumn{4}{|c|}{ Frecuencias $(\mathrm{n}=11)$} & \multirow{2}{*}{ IL1 } & \multicolumn{4}{|c|}{ Frecuencias $(\mathrm{n}=11)$} & \multirow{2}{*}{ IL1 } \\
\hline & $\mathrm{N} 1^{*}$ & N2 & N3 & N4 & & N1 & N2 & N3 & N4 & \\
\hline $\mathrm{DC} 1^{* *}$ & 9 & 2 & 0 & 0 & -1 & 0 & 7 & 4 & 0 & $-0,3$ \\
\hline DC2 & 6 & 4 & 0 & 1 & $-0,8$ & 0 & 4 & 7 & 0 & 0,3 \\
\hline DC3 & 4 & 5 & 2 & 0 & $-0,6$ & 0 & 0 & 7 & 4 & 1 \\
\hline DC4 & 6 & 3 & 2 & 0 & $-0,6$ & 0 & 0 & 10 & 1 & 1 \\
\hline DC5 & 3 & 8 & 0 & 0 & -1 & 2 & 5 & 4 & 0 & $-0,3$ \\
\hline
\end{tabular}

${ }^{*} \mathrm{~N}$ : Nivel alcanzado en cada dimensión competencial.

** DC1: Análisis cualitativo de la situación problemática; DC2: Emisión de hipótesis; DC3: Diseño de la experimentación y/o estrategia de resolución; DC4: Desarrollo de la experimentación y/o resolución del problema, y DC5: Análisis de los resultados.

Para clarificar en la medida de lo posible estos resultados y poder hacer un análisis más detallado, en la figura 2 se analiza la evolución del IL1 durante las dos situaciones problemáticas.

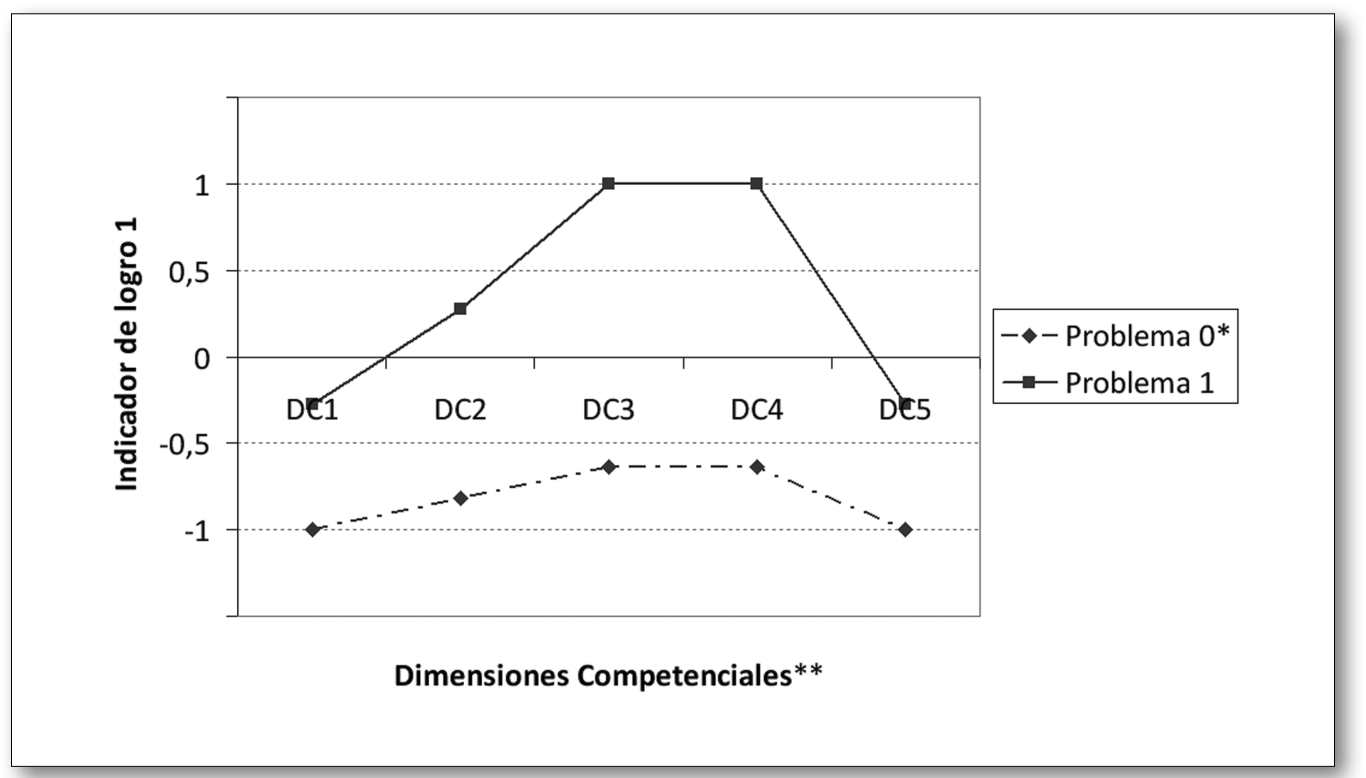

* Problema 0: «¿Qué papel absorbe más?»; problema 1: «¿Cómo separo una mezcla de hierro, cobre, corcho y sal?». ** DC1: Análisis cualitativo de la situación problemática; DC2: Emisión de hipótesis; DC3: Diseño de la experimentación y/o estrategia de resolución; DC4: Desarrollo de la experimentación y/o resolución del problema, y DC5: Análisis de los resultados.

Fig. 2. Evolución del IL1 en los problemas 0 y 1 
A partir de los resultados expuestos en la figura anterior, se puede señalar que, claramente, la primera situación problemática trabajada, realizada sin seguir una metodología concreta, muestra valores negativos del IL1 para todas las dimensiones de la competencia científica consideradas, mientras que el siguiente problema, resuelto con la MRPI, registra en la mayoría de los casos valores del IL1 positivos. En cuanto a los resultados para cada dimensión competencial:

- DC1: Análisis cualitativo de la situación problemática. El IL1 registra un valor de -1 en la primera situación problemática trabajada sin metodología expresa, o problema cero, para este primer aspecto de la competencia científica. Ello significa que ni uno solo de los alumnos de la muestra estudiada fue capaz de alcanzar un nivel 3 o 4, niveles considerados como adecuados desde un punto de vista del desarrollo competencial. Para el siguiente problema, trabajado ya siguiendo la MRPI, se observa que a pesar de que el valor del IL1 sigue siendo negativo, es muy próximo a cero. De hecho, si se consideran las frecuencias de la tabla 6, ningún alumno alcanzó el nivel 4, considerado óptimo, pero tampoco ningún alumno se quedó en el nivel 1, correspondiente al menor nivel de desarrollo competencial. Por tanto, a la vista de los datos extraídos de la investigación, se concluye que el análisis de la situación problemática por parte de los alumnos ha experimentado una mejora con la introducción de la MRPI.

- DC2: Emisión de hipótesis. En este aspecto y para el problema cero se obtiene un valor del IL1 negativo. Al menos uno de los once alumnos es capaz de alcanzar uno de los niveles más altos, pero el resto se sigue situando en los niveles 1 y 2 de menor desarrollo de la competencia. Sin embargo, en el problema 1 trabajado mediante la MRPI el valor del IL1 pasa a ser positivo, lo que quiere decir que el número de alumnos en los niveles superiores 3 y 4 es mayor que el de los alumnos en los niveles inferiores, lo que indica un buen resultado debido a la introducción de la MRPI para el desarrollo de este aspecto competencial.

- DC3: Diseño de la experimentación y/o estrategia de resolución. En esta dimensión se observa uno de los mejores resultados con la introducción de la MRPI. Mientras que en el problema cero más del $82 \%$ de los alumnos se encuentran en los niveles inferiores 1 y 2 , en el problema 1 el $100 \%$ de los alumnos son capaces de alcanzar los niveles 3 y 4 , de óptimo desarrollo competencial, y, por tanto, originan unos resultados inmejorables en su adquisición.

- DC4: Desarrollo de la experimentación y/o resolución del problema. Al igual que en el aspecto anterior, el resultado del IL1 en el problema 1 trabajado siguiendo la MRPI es el valor máximo +1 , mientras que el valor del indicador para el problema cero es negativo. Por tanto, los datos ponen de manifiesto que el uso de la MRPI también produce resultados muy relevantes en el desarrollo competencial relativo a la resolución de las situaciones problemáticas.

- DC5: Análisis de los resultados. La última dimensión analizada también presenta una evolución positiva con la introducción de la MRPI, pues pasa de un valor de indicador - 1 en el problema cero a un valor de $-0,3$ en el problema 1 . Por tanto, a pesar de que el problema resuelto con la MRPI no se sitúa en valores positivos del IL1, sí se contempla una mejora notable en el desarrollo competencial.

En definitiva, y como respuesta a esta primera pregunta de investigación, cabe concluir que en todos los aspectos de la competencia científica aquí analizados se registra una mejora, en algunos casos muy considerable, en los niveles competenciales de los alumnos con la introducción de una metodología inductiva como la MRPI. 


\section{Segunda pregunta de investigación}

Con esta segunda pregunta, «¿El uso de la MRPI permite alcanzar mayores niveles competenciales a alumnos con necesidades especiales de aprendizaje?», se desea conocer si la utilización de la MRPI para la unidad didáctica centrada en problemas implica una mejora aún más favorable en el desarrollo de la competencia científica.

Para ello, tal y como se indica en el apartado de Metodología, se considera el IL2 obtenido como el valor medio alcanzado por dimensión competencial. En la tabla 7 se muestran los valores obtenidos para dicho indicador en los problemas 1 y 2 y en la figura 3 la gráfica de los resultados.

Tabla 7.

IL2 para los problemas 1 y 2

\begin{tabular}{|c|c|c|c|c|c|c|c|c|c|c|}
\hline \multirow{3}{*}{ 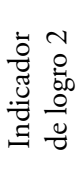 } & \multicolumn{10}{|c|}{ Situaciones Problemáticas } \\
\hline & \multicolumn{5}{|c|}{$\begin{array}{l}\text { 1. ¿Cómo separo una mezcla de hierro, } \\
\text { cobre, corcho y sal? }\end{array}$} & \multicolumn{5}{|c|}{$\begin{array}{l}\text { 2. ¿Qué hace que un pan sea más } \\
\text { esponjoso? }\end{array}$} \\
\hline & $\mathrm{DC}^{*}{ }^{*}$ & DC2 & DC3 & DC4 & DC5 & DC1 & DC2 & DC3 & DC4 & DC5 \\
\hline IL2 & 2,4 & 2,6 & 3,4 & 3,1 & 2,2 & 2,5 & 3,6 & 3,6 & 3,6 & 3,4 \\
\hline
\end{tabular}

* DC1: Análisis cualitativo de la situación problemática; DC2: Emisión de hipótesis; DC3: Diseño de la experimentación y/o estrategia de resolución; DC4: Desarrollo de la experimentación y/o resolución del problema, y DC5: Análisis de los resultados.

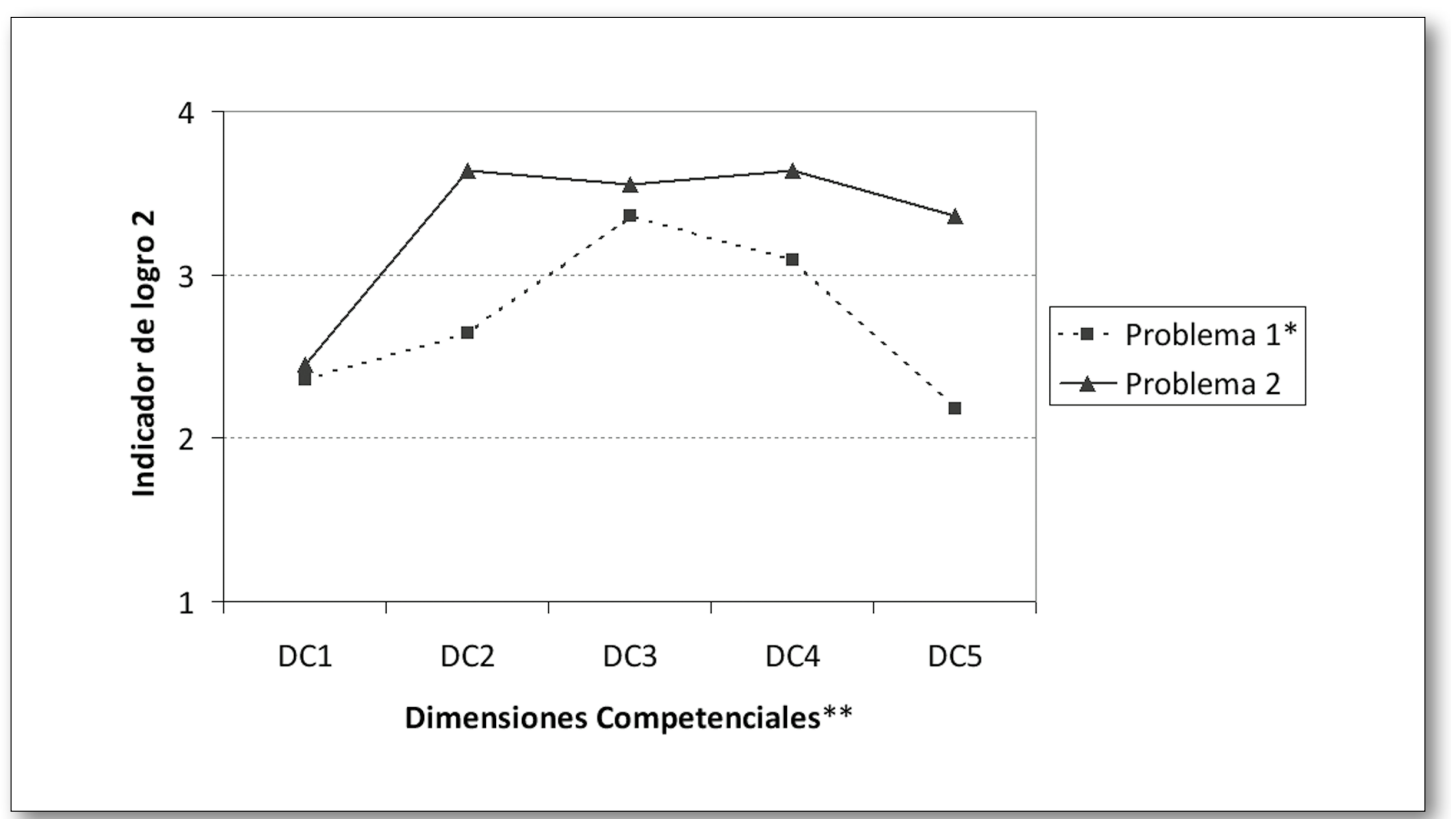

* Problema 1: ¿Cómo separo una mezcla de hierro, cobre, corcho y sal?; problema 2: ¿Qué hace que un pan sea más esponjoso?

** DC1: Análisis cualitativo de la situación problemática; DC2: Emisión de hipótesis; DC3: Diseño de la experimentación y/o estrategia de resolución; DC4: Desarrollo de la experimentación y/o resolución del problema, y DC5: Análisis de los resultados.

Fig. 3. Valor del IL2 en los problemas 1 y 2. 
A la vista de la gráfica se observa que, en promedio, los niveles de desarrollo para los distintos aspectos de la competencia son más elevados en el problema 2 que en el problema 1. Tanto en DC1 como en DC3, a pesar de que existe cierta mejoría, esta se puede considerar despreciable, pero no así en DC2, DC4 y DC5, donde el valor del IL2 es sensiblemente mayor en el problema 2 con respecto al problema 1. Por tanto, parece que el uso de la MRPI no solo es beneficioso a la hora de desarrollar la competencia científica por parte de los alumnos, como se justifica con la primera pregunta de investigación de este estudio, sino que seguir utilizando la metodología permite alcanzar niveles competenciales más elevados. Para apreciar de forma más intuitiva la evolución entre los problemas se presentan en la figura 4 unos diagramas cualitativos tipo Bliss donde el signo + indica que los escolares han experimentado un avance en el nivel de respuesta del primer al segundo problema usando la MRPI.

Por consiguiente, se puede concluir que se alcanzan niveles de desarrollo competencial cada vez más altos con el uso de la MRPI.

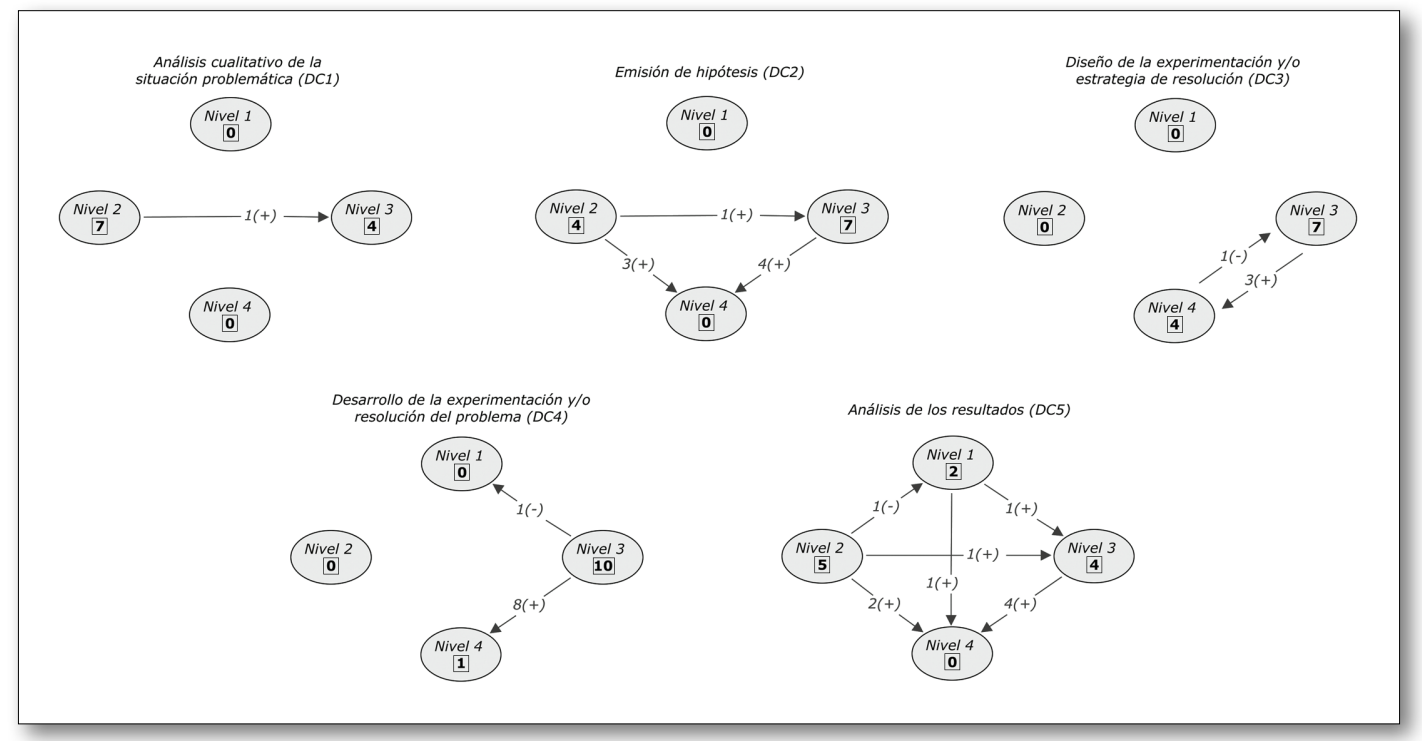

En el cuadrado de cada elipse se muestra el número de alumnos para cada nivel del problema 1, «¿Cómo separo una mezcla de hierro, cobre, corcho y sal?», mientras que los signos + o - marcan el número de alumnos que han mejorado o empeorado de nivel, respectivamente, para el problema 2, "¿Qué hace que un pan sea más esponjoso?» Fig 4. Diagramas de Bliss con la evolución de las DC entre el problema 1, «¿Cómo separo una mezcla de hierro, cobre, corcho y sal?», y el problema 2, «¿Qué hace que un pan sea más esponjoso?»

\section{Tercera pregunta de investigación}

La última pregunta planteada, «¿En qué medida satisface a los alumnos del ACT el uso de un método indagativo como la Metodología de Resolución de Problemas como Investigación?», iba en la línea de analizar el nivel de complacencia de los alumnos respecto al método en sí, aspecto muy relevante para un programa de diversificación. Para ello, se ha adaptado un cuestionario con doce preguntas o ítems, utilizado por Varela (1994) y también por Ibáńez (2003), ya caracterizado en la tabla 4, y que se presenta en el anexo IV. Como se observa, también se solicitaba un por qué con la intención de que los estudiantes pudieran manifestar sus inquietudes y aportasen información secundaria. En la tabla 8 se muestran en tanto por ciento los resultados obtenidos. 
Tabla 8.

Resultados obtenidos en el cuestionario de satisfacción de los alumnos hacia la MRPI

\begin{tabular}{|c|r|r|r|r|r|}
\hline \multirow{2}{*}{ Items } & \multicolumn{5}{|c|}{ Valoración de las respuestas en \% } \\
\cline { 2 - 6 } & 1 & 2 & 3 & 4 & 5 \\
\hline 1 & 0 & 10 & 20 & 50 & 20 \\
\hline 2 & 0 & 20 & 50 & 20 & 10 \\
\hline 3 & 10 & 0 & 30 & 40 & 20 \\
\hline 4 & 10 & 10 & 50 & 20 & 10 \\
\hline 5 & 10 & 20 & 40 & 30 & 0 \\
\hline 6 & 10 & 30 & 30 & 20 & 10 \\
\hline 7 & 0 & 0 & 30 & 30 & 40 \\
\hline 8 & 0 & 30 & 30 & 20 & 20 \\
\hline 9 & 10 & 10 & 30 & 20 & 30 \\
\hline 10 & 20 & 30 & 30 & 10 & 10 \\
\hline 11 & 0 & 10 & 20 & 40 & 30 \\
\hline 12 & 10 & 0 & 40 & 40 & 10 \\
\hline
\end{tabular}

A continuación se analizan las respuestas en función de las cuatro categorías indicadas en la tabla 4 en que se agrupan los ítems.

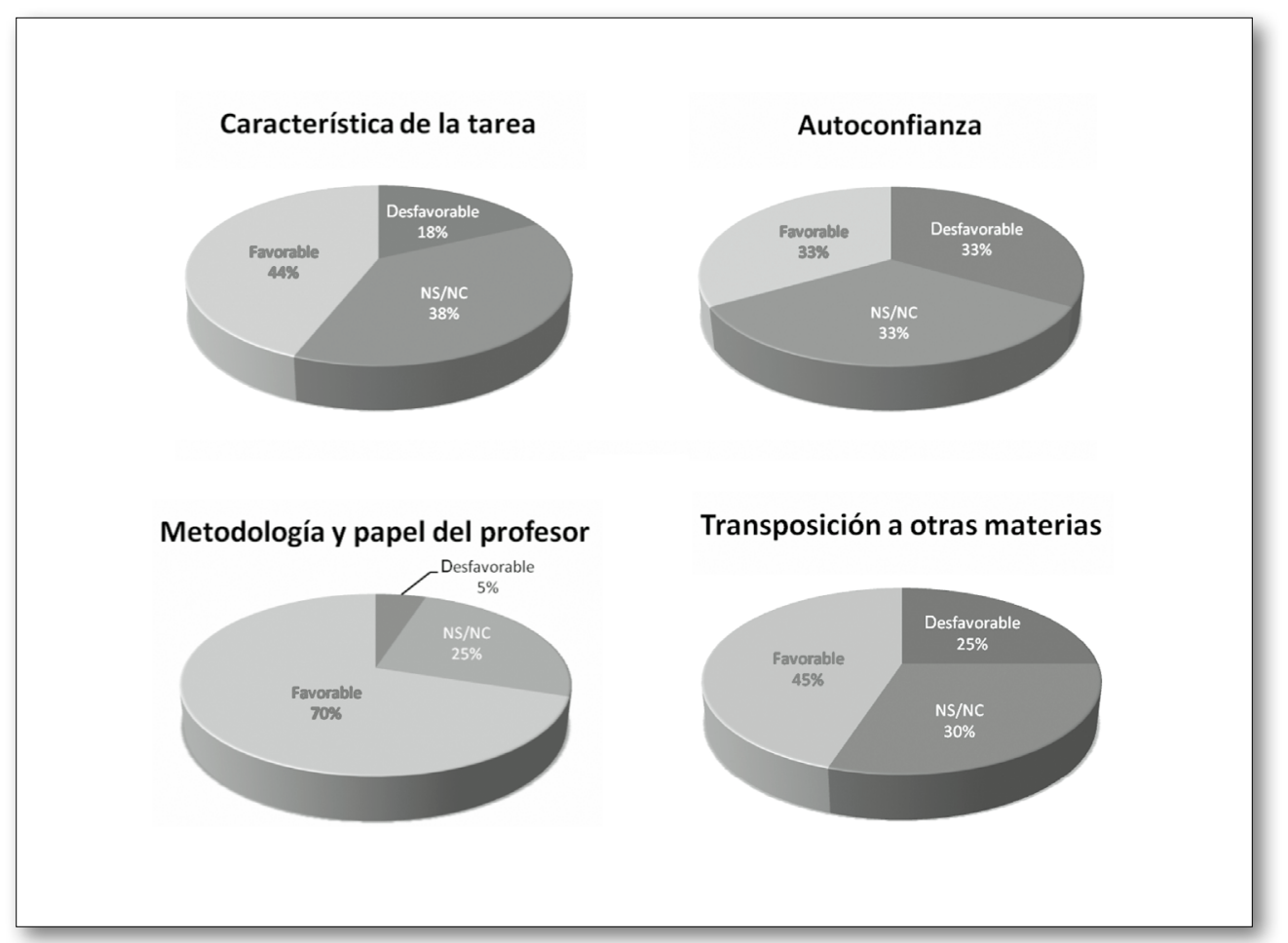

Fig. 5. Diagramas referentes a la satisfacción de los alumnos frente a la MRPI por categorías.

Lo primero que llama la atención es que el número de alumnos que se sitúan en una puntuación 3 de «no sabe/no contesta» es elevado, del orden de un tercio, en las cuatro categorías. En los ítems 
relacionados con la característica de la tarea y con la transposición a otras materias, los estudiantes que sí se decantan hacia uno de los dos extremos son en torno al doble los que ofrecen una respuesta favorable de los que realizan una aportación desfavorable. En los ítems concernientes con la metodología y el papel del profesor es donde se aprecia un número de respuestas favorables más destacable. Aquí, los alumnos no solo agradecen que el profesor se muestre atento y los oriente durante el proceso, sino que también valoran muy positivamente que se utilice una metodología que les permita pensar por ellos mismos, lo cual es algo digno de mención.

Para tratar de tener una visión más general de la satisfacción hacia la MRPI del grupo de diversificación con el que se ha trabajado, se han calculado los valores medios sobre el total de ítems, agrupando las respuestas 1 y 2 de la escala de Likert como puntuaciones negativas y las respuestas 4 y 5 como puntuaciones positivas. Así, un $21 \%$ de los alumnos manifiestan, de media, puntuaciones negativas a las preguntas del cuestionario, frente a un $46 \%$ que las puntúan de manera positiva. Los datos no son tan buenos como los obtenidos por Varela (1994) para Física, o por Ibáñez (2003) para Biología, pero aun así, la valoración hacia la MRPI de los alumnos de la muestra analizada es favorable.

Del análisis individualizado de cada ítem no se puede extraer ningún rasgo especialmente negativo. Lo más destacable son los individuos que puntualmente ofrecen una calificación baja, justificada por el alto grado de esfuerzo que les supone la MRPI o por su pobre interés hacia las ciencias. También son dignos de mención los datos obtenidos para puntuaciones positivas: en los ítems relativos a la característica de la tarea (cuestiones 1, 2, 3, 4 y 5), entre un 30 y un 70\% de los alumnos ofrecen puntuaciones positivas, frente a un intervalo del 10 al 30\% cuyas respuestas son negativas; los ítems que hacen referencia a la metodología y al papel del profesor (cuestiones 7 y 11 ) indican un $70 \%$ de respuestas positivas frente a un $5 \%$, de media, de respuestas negativas, y los ítems vinculados a la transferencia a otras materias (cuestiones 8 y 9) manifiestan unas medias del $45 \%$ de respuestas positivas frente a un $25 \%$ negativas.

Dicho lo anterior, a partir de los datos obtenidos se concluye que los alumnos de la muestra presentan una actitud favorable y un alto grado de satisfacción ante la resolución de situaciones problemáticas abiertas utilizando la MRPI.

\section{CONCLUSIONES}

En vista de los resultados del estudio, se puede afirmar que la MRPI presenta, de manera general, unos buenos resultados en el desarrollo de la competencia cientifica en diversificación.

En relación con la primera pregunta de investigación:

1. Los estudiantes de diversificación han experimentado una mejora sustancial en los diferentes aspectos de las dimensiones de la competencia científica tras la introducción de la MRPI, especialmente en los aspectos relacionados con la experimentación, tanto en la planificación (DC3: Diseño de la experimentación y/o estrategia de resolución) como en la puesta en marcha de esta (DC4: Desarrollo de la experimentación y/o resolución del problema). La mejora también es relevante en relación con el planteamiento de soluciones tentativas (DC2: Emisión de hipótesis), y, en menor grado, respecto al análisis del problema y de los resultados (DC1: Análisis cualitativo de la situación problemática, y DC5: Análisis de los resultados).

Respecto a la segunda pregunta de investigación:

2. La utilización de la MRPI para los problemas de la unidad didáctica ha permitido a alumnos con necesidades especiales de aprendizaje alcanzar niveles competenciales mayores, fundamentalmente en las dimensiones DC2: Emisión de hipótesis, y DC5: Análisis de los resultados, y, en menor medida, en DC4: Desarrollo de la experimentación y/o resolución del problema. 
En relación con la tercera pregunta de investigación:

3. Los alumnos del ámbito científico-tecnológico han mostrado una actitud favorable respecto al uso de la MRPI para la resolución de situaciones problemáticas. Un 46\% de los estudiantes realizan aportaciones positivas, frente a un $21 \%$ que manifiestan una predisposición negativa, justificada esta más por el nivel de esfuerzo que implica la metodología o su escasa predilección por las ciencias que por razones en contra del método en sí mismo.

Como recapitulación, podemos afirmar que los alumnos de diversificación objeto del estudio han desarrollado, de manera progresiva, niveles de competencia más altos con la introducción y uso de la MRPI para resolver situaciones problemáticas, manifestando, además, un grado de satisfacción notable con ello.

\section{AGRADECIMIENTOS}

Nuestro agradecimiento a los escolares y a D. Agustín Criado, subdirector de Coordinación Pedagógica del Colegio Lourdes, por su inestimable colaboración.

\section{REFERENCIAS BIBLIOGRÁFICAS}

Abd-El-Khalick, F.; Boujaoude, S.; Duschl, R.; Lederman, N.G.; Mamlok-Naaman R.; Hofstein, A.; Treagust, D y Tuan, H-L. (2004). Inquiry in Science Education: International perspective. Science Education, 88, pp. 397-419.

http://dx.doi.org/10.1002/sce.10118

Anderson, R.D. (2002). Reforming science teaching: What research says about inquiry? Journal of Science Teacher Education, 13(1), pp. 1-12. http://dx.doi.org/10.1023/A:1015171124982

APU (Assessment of Performance Unit) (1987). Assessing investigations at ages 13 and 15. Science report for teachers 9. Letchworth: Adiard\&Son Ltd. The Garden City Press.

Boyano SÁnchez, F.J. (2000). El Teñido. Problema abierto para el ámbito científico-tecnológico. Cuadernos de Pedagogía, 293, pp. 87-89.

Bransford, J.D.; Brown, A.L. y Cocking, R.R. (2000). How People Learn: Brain, Mind, Experience and School. Washington, D.C.: Academy Press. Disponible en línea: www.nap.edu/books/0309070368/html. (Última consulta, 23-07-2013).

CM (2007). Orden 4265/2007, de 2 de agosto, de la Consejera de Educación, por la que se regula el programa de diversificación curricular en la Educación Secundaria Obligatoria de la Comunidad de Madrid.

Colburn, A. (2000). An inquiry primer. Science Scope, 23(6), pp. 42-44.

Domin, D.S. (2007). Students' perceptions of when conceptual development occurs during laboratory instruction. Chemistry Education Research and Practice, 8(2), pp. 140-152. http://dx.doi.org/10.1039/b6rp90027e

Felder, R.M. y Brent, R. (1996). Navigating the bumpy road to student-centered instruction. College Teaching, 44 (2), pp. 43-47. Disponible en línea: www.ncsu.edu/felderpublic/Papers/Resist.html. (Última consulta, 23-07-2013).

Fundación La Main À La PÂte. (2011). Disponible en línea: http://www.fondation-lamap.org. (Última consulta, 23-07-2013).

Gil, D. y Martínez-Torregrosa, J. (1983). A model for problem-solving in accordance with scientific methodology. European Journal of Science Education, 5(4), pp. 447-455.

http://dx.doi.org/10.1080/0140528830050408 
Hernández Sampieri, R.; Fernández Collado, C. y Baptista Lucio, P. (2006). Metodología de la Investigación (4th ed.). México: McGraw-Hill.

Hmelo-Silver, C.E. (2004). Problem-Based Learning: What and how do students learn? Educational Psychology Review, 16(3), pp. 235-265.

http://dx.doi.org/10.7771/1541-5015.1004

Hmelo-Silver, C.E. y Barrows, H.S. (2006). Goals and strategies of a problema-based learning facilitador. Interdisciplinary Journal of Problem-based Learning, 1, pp. 21-39.

http://dx.doi.org/10.1080/00461520701263368

Hmelo-Silver, C.E.; Duncan, R.G. y Chinn, C.A. (2007). Scaffolding and achievement in problem-based and inquiry learning: a response to Kirschner, Sweller, and Clark (2006). Educational Psychologist, 42(2), pp. 99-107.

http://dx.doi.org/10.1023/B:EDPR.0000034022.16470.f3

Hofstein, A. (2003). The laboratory in chemistry education: Thirty years of experience with developments, implementation and research. Chem. Ed. Res. Pract., 5(3), pp. 247-264.

http://dx.doi.org/10.1039/b4rp90027h

IвÁŃEZ, M. ${ }^{a}$ T. (2003). Aplicación de una metodología de resolución de problemas como una investigación para el desarrollo de un enfoque ciencia-tecnologia-sociedad en el curriculo de biología de educación secundaria. Tesis doctoral, Universidad Complutense, Madrid.

IbáNÉz, M. a T. y Martínez Aznar, M. ${ }^{a}$ M. (2005). Solving problems in genetics (II): Conceptual Restructuring. International Journal of Science Education, 27(12), pp. 1495-1519.

http://dx.doi.org/10.1080/09500690500186584

IbáŃEz, M. ${ }^{a}$ T. y Martínez Aznar, M. ${ }^{a}$ M. (2007). Solving problems in genetics (III): Change in the view of the nature of science. International Journal of Science Education, 29(6), pp. 747-769. http://dx.doi.org/10.1080/09500690600855369

INFORME Rocard (2007). Science Education now: A renewed pedagogy for the future of Europe. Bruselas: European Commision. Disponible en línea:

http://ec.europa.eu/research/science-society/document_library/pdf_06/report-rocard-on-scienceeducation_en.pdf. (Última consulta, 23-07-2013).

Johnson, D.W. y Johnson, R.T. (2008). Cooperation and the use of technology. En J.M. Spector, M.D. Merrill, J.E. Van Merriënboer y M.P. Driscoll (eds.). Handbook of research on educational communications and technology. Third Edition. New York: Lawrence Earlbaum Associates (LEA), Taylor \& Francis Group, pp. 402-418.

http://dx.doi.org/10.1177/0010836708092837

Keys, C.W. y Bryan, L.A. (2001). Co-constructing Inquiry-Based Science with teachers: essential research for lasting reform. Journal of Research in Science Teacher, 38(6), pp. 631-645. http://dx.doi.org/10.1002/tea.1023

Linn, M.C., Davis, E.A. y Bell, P. (2004). Internet environments for science education. Mahwah: Lawrence Erlbaum Associates.

Martínez Aznar, M. ${ }^{a}$ M. e IbáŃEz, M. ${ }^{a}$ T. (2005). Solving problems in genetics. International Journal of Science Education, 27(1), pp. 101-121.

http://dx.doi.org/10.1080/09500690410001673801

Martínez Aznar, M. ${ }^{a}$ M. (2009). La MRPI una metodología investigativa para el desarrollo de las competencias científicas escolares en la Educación Primaria. En J. Cervelló (coord.). Educación cientifica "ahora»: el informe Rocard. Madrid: MEC, Subdirección General de Información y Publicaciones, pp. 47-78.

Martínez Aznar, M. a M. y Bárcena Martín, A.I. (2013). ¿Es beneficioso masticar bien para realizar una buena digestión? Una actividad de indagación en un aula de diversificación. Educació Química, EduQ, 14 (2013), pp. 19-28. 
Martínez Aznar, M. ${ }^{a}$ M. e IbáÑEz, M. ${ }^{\text {T T. }}$ (2006). Resolver situaciones problemáticas en genética para modificar las actitudes relacionadas con la ciencia. Enseñanza de las Ciencias, 24(2), pp. 193-206.

Martínez Aznar, M. a M. y Ovejero, P. (1997). Resolver el problema abierto: «Teñir lanas a partir de productos colorantes naturales». Una actividad investigativa para la enseñanza obligatoria. Enseñanza de las Ciencias, 15(3), pp. 401-422.

Martínez Aznar, M. a M. y Varela, M. a P. (1996). De la resolución de problemas al cambio conceptual. Investigación en la Escuela, 28, pp. 69-78.

Martínez Aznar, M. ${ }^{a}$ M. y Varela, M. ${ }^{a}$ P. (1997). Influencia de las diferencias individuales en la resolución de problemas abiertos de Física. En Beltrán (ed). Nuevas perspectivas en la intervención psicopedagógica: I. Aspectos cognitivos, motivacionales y contextuales. Madrid: UCM.

Martínez Aznar, M. ${ }^{a}$ M. y Varela, M. ${ }^{a}$ P. (2009). La resolución de problemas de energía en la formación inicial de maestros. Enseñanza de las Ciencias, 27(3), pp. 343-360.

MEC (2006). Ley Orgánica 2/2006, de 3 de mayo, de Educación.

MEC (2007). R.D. 1631/2006, de 29 de diciembre, por el que se establecen las enseñanzas mínimas correspondientes a la Educación Secundaria Obligatoria.

Mohedano, M. J. (2000). Programas de diversificación curricular. Aciertos y dudas. Cuadernos de Pedagogía, 293, pp. 23-27.

Prince, M.J. y Felder, R.M. (2006). Inductive Teaching and Learning Methods: Definitions, Comparisons and Research Bases. Journal Engineering Education, 95(2), pp. 123-138. http://dx.doi.org/10.1002/j.2168-9830.2006.tb00884.x

Prince, M.J. y Felder, R.M. (2007). The Many Faces of Inductive Teaching and Learning. Journal of College Science Teaching, 36(5), pp. 14-20. National Science Teachers Association (NSTA).

Proyecto Primas. (2013) Disponible en línea: http://www.primas-project.eu. (Última consulta, 1811-2013).

Quintanilla, M.; Joglar, C.; Jara, R.; Camacho, J.; Ravanel, E.; Lavamere, A.; Cuellar, L.; IzQuierdo, M. y Chamizo, J. (2010). Resolución de problemas científicos escolares y promoción de competencias de pensamiento científicos. ¿Qué piensan los docentes de química en ejercicio? Enseñanza de las Ciencias, 28(2), pp. 185-198.

Ramírez, J.L., Gil, D. y Martínez-Torregrosa, J. (1994). La resolución de problemas de física y quimica como investigación. Madrid: MEC.

Rodríguez Gómez, G.; Gil Flores, J. y García Jiménez, E. (1996). Metodología de la Investigación Cualitativa. Granada: Ediciones Aljibe.

Schwartz, R.S.; Lederman, N.G. y Crawford, B.A. (2004). Developing views of nature of science in an authentic context: an explicit approach to bridging the gap between nature of science and scientific inquiry. Science Education, 88(4), pp. 610-645.

http://dx.doi.org/10.1002/sce.10128

Torrego, L. y Leal, P. (2009). Estudio evaluativo de casos sobre el ámbito científico y tecnológico de los programas de diversificación curricular. Educación y Diversidad, 3, pp. 195-215.

Tortosa, M. (2013). Aprendizaje sobre disoluciones reguladoras de $\mathrm{pH}$ mediante indagación guiada utilizando sensores. Enseñanza de las Ciencias, 31(1), pp. 189-211.

Varela, M. a P. (1994). La resolución de problemas en la enseñanza de las ciencias. Aspectos didácticos y cognitivos. Tesis doctoral, Universidad Complutense, Madrid.

Varela, M. a P. y Martínez Aznar, M. ${ }^{a}$ M. (1997). Una estrategia de cambio conceptual en la enseñanza de la Física: La resolución de problemas como actividad de investigación. Enseñanza de las Ciencias, 15(2), pp. 173-188.

Verger, S. (2001). Los programas de diversificación curricular en la Educación Secundaria Obligatoria: el difícil equilibrio entre la comprensividad y la atención a la diversidad. Tesis doctoral, Universidad de las Islas Baleares, Palma de Mallorca. 


\title{
ANEXO 1
}

Plantilla utilizada en diversificación para resolver situaciones problemáticas abiertas siguiendo la MRPI (Martínez Aznar, 2009)

\section{VOY A TRABAJAR COMO LOS CIENTÍFICOS. PASOS QUE SEGUIR}

\section{EL PROBLEMA}

\section{Análisis del problema}

1.1 ¿Qué me están pidiendo? - Reformulación en términos operativos

1.2 ¿Qué necesito saber? - Marco teórico de referencia

1.3 ¿Qué voy a considerar y qué no? - Restricción de condiciones

2. Emisión de hipótesis

2.1¿Qué va a suceder? - Hipótesis que relacionen los factores implicados

\section{BÚSQUEDA DE LA SOLUCIÓN}

3. Estrategia para resolver el problema paso a paso

3.1 ¿Qué cosas cambian y cuáles no cambian? - Variables

3.2 ¿Qué voy a medir y qué materiales necesito?

3.3 Voy a dibujar lo que quiero hacer

3.4 ¿He hecho antes algo parecido? - Analogías

3.5 Ahora decido qué voy a hacer paso a paso - Toma de decisiones

4. Desarrollo de los experimentos

4.1 Explico los pasos que he seguido

4.2 ¿De qué me he dado cuenta?, ¿qué he observado?

4.3 ¿Qué medidas he tomado?, ¿qué datos he obtenido?

\section{ANÁLISIS DEL PROBLEMA}

5. Análisis de los resultados

5.1 ¿Siempre ocurre lo mismo? - Regularidades

5.2 ¿A qué conclusiones he llegado?

5.3 ¿Los resultados respaldan mi hipótesis?

\begin{abstract}
ANEXO 2
Aspectos fundamentales para la resolución de la situación problemática «¿Qué hace que un pan sea más esponjoso?»

- DC1. Análisis cualitativo de la situación problemática

En este apartado habrá que tener en cuenta una serie aspectos como: 1.1) Reformular el problema como el análisis de los factores que hacen que se produzca mayor cantidad de $\mathrm{CO}_{2}$ en la fermentación del pan. 1.2) Definir los componentes básicos del pan, como harina, agua, levadura y sus características principales. 1.3) Enumerar las fases en la producción del pan, como la elaboración de la masa, su reposo, el horneado y el enfriado, así como en qué consiste cada una de ellas. 1.4) Restringir la solución del problema a la utilización de un solo tipo de levadura.
\end{abstract}




\section{- DC2. Emisión de hipótesis}

Enunciados planteados de manera similar a: 2.1) La cantidad de levadura en la masa influye en el volumen de $\mathrm{CO}_{2}$ producido. 2.2) El tiempo de amasado influye en el volumen de $\mathrm{CO}_{2}$ producido. 2.3) La temperatura de reposo de la masa influye en el volumen de $\mathrm{CO}_{2}$ producido.

- DC3. Diseño de la experimentación y/o estrategia de resolución

Partiendo de la hipótesis 2.1 definida por la cantidad de levadura en la masa, habrá que considerar: 3.1) La necesidad de utilizar, a parte de los ingredientes básicos, otros utensilios o sustancias como recipientes diversos, sal, cucharas, una balanza, un cronómetro, etc. 3.2) Se explicarán paso a paso las decisiones tomadas para resolver el problema y se indicará el proceso de manera esquemática. A modo de ejemplo:

Echa media cucharadita de levadura en un cuenco con $100 \mathrm{ml}$ de agua. Deja durante 5 minutos y después revuelve para que se disuelva. Mezcla la harina junto a la sal en un recipiente grande. Haz un hoyo en el centro, vierte el agua con la levadura y mezcla. Añade el resto del agua, según sea necesario, hasta formar una masa firme y húmeda. Tapa con un paño de cocina y deja reposar 20 minutos hasta que la masa esponje, adquiera una consistencia suave y aumente ligeramente de volumen. Echa la masa sobre una superficie ligeramente enharinada. Amasa aproximadamente 10 minutos, hasta obtener una masa homogénea, brillante y elástica. Pon la masa en un recipiente limpio y tapa con un paño de cocina. Deja fermentar entre 1,5 y 2 horas, hasta que doble el tamaño. Introduce la masa en un vaso de precipitados de $400 \mathrm{ml}$ previamente enharinado. Cuece en el horno, previamente calentado, a $180^{\circ} \mathrm{C}$ durante 30 minutos, hasta que el pan adquiera un color dorado. Deja que el pan horneado se enfríe. Repite el proceso echando una cucharadita y media de levadura para poder comparar cuál de los dos panes queda más esponjoso.

3.4) Se indicará si es posible alguna analogía con un problema previo.

- DC4. Desarrollo de la experimentación y/o resolución del problema

Aquí se tendrá en cuenta: 4.1) Explicar paso a paso la experimentación realizada, como por ejemplo:

En $5 \mathrm{ml}$ de agua templada disolvimos $1 / 4$ cucharadita de levadura en polvo utilizando un vaso de precipitados. En otro vaso de precipitados mezclamos $25 \mathrm{~g}$ de harina y $1 / 2$ cucharadita de sal y añadimos el agua con la levadura disuelta. Removimos ańadiendo otros $5 \mathrm{ml}$ de agua hasta obtener una masa uniforme. Dejamos reposar 20 minutos tapando la masa con papel absorbente. Sobre una superficie enharinada trabajamos la masa 10 minutos. Después de amasar, introducimos la masa en un vaso de precipitados y lo tapamos con papel absorbente. Dejamos fermentar 1,5 horas. Introducimos la masa en un vaso de precipitados de $250 \mathrm{ml}$ previamente enharinado y horneamos a $180^{\circ} \mathrm{C}$ durante unos 30 minutos. Repetimos el proceso pero con el doble de levadura.

4.2) Se indicará si es posible alguna observación relevante, como por ejemplo: «antes de hornear ya se aprecia mayor volumen en el pan que tiene más cantidad de levadura».

- DC5. Análisis de los resultados

En este aspecto se valorará: 5.1) El registro de regularidades en los datos como «todos los panes elaborados con mayor cantidad de levadura tienen más volumen». 5.2) Que las conclusiones estén fundamentadas en los datos obtenidos y relacionadas con la hipótesis de partida, por ejemplo, «a mayor cantidad de levadura en la masa, obtenemos un pan más esponjoso y por tanto la hipótesis es correcta». 


\section{ANEXO 3}

Criterios de corrección según niveles para las variables de la situación problemática 2 «¿Qué hace que mi pan sea más esponjoso?»

\begin{tabular}{|c|c|c|}
\hline $\begin{array}{l}\text { Dimensión } \\
\text { competencial }\end{array}$ & Nivel & Descripción \\
\hline \multirow{4}{*}{ DC1 } & 1 & No contestan o sus aportaciones no son relevantes. \\
\hline & 2 & Enuncian algunos ingredientes para la elaboración del pan. \\
\hline & 3 & Definen los ingredientes y el procedimiento (la receta) para la elaboración del pan. \\
\hline & 4 & $\begin{array}{l}\text { Definen los ingredientes necesarios para la elaboración del pan, sus propiedades, la receta y el } \\
\text { proceso de fermentación. }\end{array}$ \\
\hline \multirow{4}{*}{ DC2 } & 1 & No contestan o sus aportaciones no son relevantes. \\
\hline & 2 & $\begin{array}{l}\text { Plantean una hipótesis de manera implícita pero incoherente, dado que su comprobación no } \\
\text { conduciría a la resolución del problema. }\end{array}$ \\
\hline & 3 & $\begin{array}{l}\text { Plantean un enunciado coherente con la situación problemática pero no lo explicitan en forma } \\
\text { de hipótesis. }\end{array}$ \\
\hline & 4 & Plantean una hipótesis explícita y coherente con el problema. \\
\hline \multirow{4}{*}{ DC3 } & 1 & No contestan o sus aportaciones no son relevantes. \\
\hline & 2 & $\begin{array}{l}\text { Planifican una estrategia errónea que no conduce a la resolución del problema o que tiene algún } \\
\text { fallo de diseño. }\end{array}$ \\
\hline & 3 & Planifican una estrategia correcta pero no explicitan su desarrollo. \\
\hline & 4 & $\begin{array}{l}\text { Explican paso a paso el proceso que van a seguir para resolver el problema, lo representan gráfi- } \\
\text { camente y definen materiales necesarios. }\end{array}$ \\
\hline \multirow{4}{*}{ DC4 } & 1 & No contestan o sus aportaciones no son relevantes. \\
\hline & 2 & Realizan una experimentación caótica y sin un objetivo claro. \\
\hline & 3 & Exponen ordenadamente el desarrollo de su experimentación pero no aportan observaciones. \\
\hline & 4 & $\begin{array}{l}\text { Exponen ordenadamente el desarrollo de su experimentación y ponen de manifiesto las posibles } \\
\text { variaciones respecto a lo planificado. Aportan observaciones cualitativas y/o cuantitativas que } \\
\text { hayan despertado su interés o curiosidad. }\end{array}$ \\
\hline \multirow{4}{*}{ DC5 } & 1 & No contestan o sus aportaciones no son relevantes. \\
\hline & 2 & Su análisis se limita a exponer los datos obtenidos. \\
\hline & 3 & Razonan conclusiones correctas pero sin apoyarse en datos que las respalden. \\
\hline & 4 & $\begin{array}{l}\text { Llegan a conclusiones coherentes razonando a partir de los datos obtenidos y relacionan estas } \\
\text { con la hipótesis de partida. }\end{array}$ \\
\hline
\end{tabular}

DC1: Análisis cualitativo de la situación problemática. DC2: Emisión de hipótesis. DC3: Diseño de la experimentación y/o estrategia de resolución. DC4: Desarrollo de la experimentación y/o resolución del problema. DC5: Análisis de los resultados. 


\section{ANEXO 4}

Cuestionario de satisfacción (Ibañez, 2003)

- Señala con una cruz la casilla que te corresponde:

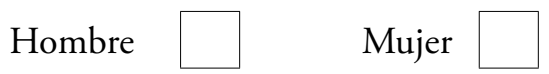

- ¿Estás interesado en continuar estudiando algo que esté relacionado con las ciencias? ¿Qué?

1. La MRPI te ha parecido una tarea (MRPI: Metodología de Resolución de Problemas como Investigación)

\begin{tabular}{|c|c|c|c|c|c|c|}
\hline \multirow{2}{*}{ Aburrida } & 1 & 2 & 3 & 4 & 5 & \multirow{2}{*}{ Interesante } \\
\cline { 2 - 6 } & & & & & & \\
\hline
\end{tabular}

Porque...

2. La MRPI te ha resultado una actividad

\begin{tabular}{|l|l|l|l|l|l|l|}
\hline \multirow{2}{*}{ Difícil } & 1 & 2 & 3 & 4 & 5 & \multirow{2}{*}{ Fácil } \\
\cline { 2 - 5 } & & & & & & \\
\hline
\end{tabular}

Porque...

3. La MRPI te ha ayudado a aprender ideas de ciencias

\begin{tabular}{|l|l|l|l|l|l|l|}
\hline \multirow{2}{*}{ Poco } & 1 & 2 & 3 & 4 & 5 & \multirow{2}{*}{ Mucho } \\
\cline { 2 - 5 } & & & & & & \\
\hline
\end{tabular}

Porque...

4. La MRPI te ha ayudado a entender cómo trabajan los científicos

\begin{tabular}{|l|l|l|l|l|l|l|}
\hline \multirow{2}{*}{ Poco } & 1 & 2 & 3 & 4 & 5 & \multirow{2}{*}{ Mucho } \\
\cline { 2 - 5 } & & & & & & \\
\hline
\end{tabular}

Porque...

5. La MRPI te va a ayudar a resolver problemas como los del libro

\begin{tabular}{|l|l|l|l|l|l|l|}
\hline \multirow{2}{*}{ Poco } & 1 & 2 & 3 & 4 & 5 & \multirow{2}{*}{ Mucho } \\
\cline { 2 - 5 } & & & & & & \\
\hline
\end{tabular}

Porque... 
6. Con la MRPI te sientes capaz de resolver otros problemas desconocidos

\begin{tabular}{|l|l|l|l|l|l|l|}
\hline \multirow{2}{*}{ No } & 1 & 2 & 3 & 4 & 5 & \multirow{2}{*}{ Sí } \\
\cline { 2 - 5 } & & & & & & \multicolumn{1}{c|}{ Sinn } \\
\hline
\end{tabular}

Porque...

7. ¿Cómo han sido los profes? ¿Su ayuda ha sido útil?

\begin{tabular}{|l|l|l|l|l|l|l|}
\hline \multirow{2}{*}{ Inútil } & 1 & 2 & 3 & 4 & 5 & \multirow{2}{*}{ Muy útil } \\
\cline { 2 - 5 } & & & & & & \\
\hline
\end{tabular}

Porque...

8. El tipo de problemas que hemos resuelto, ¿te ayudará en el futuro a resolver situaciones de tu vida cotidiana (hogar, automóvil, juegos...)?

\begin{tabular}{|l|l|l|l|l|l|l|}
\hline \multirow{2}{*}{ Poco } & 1 & 2 & 3 & 4 & 5 & \multirow{2}{*}{ Mucho } \\
\cline { 2 - 5 } & & & & & & \\
\hline
\end{tabular}

Porque...

9. ¿Crees que esta forma de trabajar es útil para problemas de otras materias: Mates, Biología...?

\begin{tabular}{|l|l|l|l|l|l|l|}
\hline \multirow{2}{*}{ Poco } & 1 & 2 & 3 & 4 & 5 & \multirow{2}{*}{ Mucho } \\
\cline { 2 - 5 } & & & & & & \\
\hline
\end{tabular}

Porque...

10. La forma en que hemos trabajado la asignatura, ¿te ha abierto nuevas perspectivas e interés sobre qué es la Física y Química o las Ciencias en general?

\begin{tabular}{|l|l|l|l|l|l|l|}
\hline \multirow{2}{*}{ No } & 1 & 2 & 3 & 4 & 5 & \multirow{3}{*}{ Sí } \\
\cline { 2 - 5 } & & & & & & \multirow{2}{*}{} \\
\hline
\end{tabular}

Porque....

11. ¿Te gusta esta manera de trabajar en clase de ACT?

\begin{tabular}{|l|l|l|l|l|l|l|}
\hline \multirow{2}{*}{ Poco } & 1 & 2 & 3 & 4 & 5 & \multirow{2}{*}{ Mucho } \\
\cline { 2 - 5 } & & & & & & \multirow{2}{*}{ Mun } \\
\hline
\end{tabular}

Porque...

12. ¿Ahora te sientes más seguro para resolver problemas?

\begin{tabular}{|l|l|l|l|l|l|l|}
\hline \multirow{2}{*}{ Poco } & 1 & 2 & 3 & 4 & 5 & \multirow{2}{*}{ Mucho } \\
\cline { 2 - 5 } & & & & & & \\
\hline
\end{tabular}

Porque... 


\title{
The methodology of problem-solving as an investigation (MPSI): an inquiry approach for developing the scientific competence in diversification program students
}

\author{
Fernando Pavón Martínez \\ fpavon@ucm.es \\ M. ${ }^{a}$ Mercedes Martínez Aznar \\ Departamento de Didáctica de las Ciencias Experimentales, Facultad de Educación-Centro de Formación del Profe- \\ sorado. Universidad Complutense, Madrid \\ mtzaznar@ucm.es
}

Different studies reveal the advantages of IBSE (Inquiry-based Science Education) methods for science teaching, but unfortunately these are not the most common in classrooms. The Spanish law LOE, article 27, indicates that secondary education requires a specific methodology that involves contents organization and practice activities different from those generally established, something essential in diversification programs, where traditional methodologies based on teacher's explanation do not work. The students characteristics in these programs lead us to predict that the use of an IBSE method such as the Methodology of Problem-Solving as an Investigation (MPSI) may encourage the construction of knowledge included in the scientific and technological field. Therefore, in this paper we analyse the use of this methodology in the learning sequence Physical changes and chemical changes, in a diversification program, and study the efficacy of this methodology for the development of scientific competence.

Thus, we consider the following questions:

1. Does the introduction of Methodology of Problem-Solving as an Investigation help diversification students to evolve in the development of scientific competence?

2. Does using the Methodology of Problem-Solving as an Investigation allow achieving higher levels of competence to students with special learning needs?

3. To what extent are diversification students satisfied with using an inquiry method as the Methodology of Problem-Solving as an Investigation?

First of all, in order to make a detailed study of scientific competence, the phases of the MPSI are correlated with the following dimensions of competence: a) the problem situation analysis, b) the hypothesis emission, c) the problem solving or experimentation design, d) the experimentation development, and e) the results analysis. This way we can analyse the solutions written by the students and study the development of the competence dimensions.

We have worked with a group of eleven students in the scientific and technological field in the 4th year of Secondary Education in a diversification program in a school of Madrid. These students have, in general, learning disabilities, impaired social relationships, low self-esteem and motivation, and more than half of them also have problems with the language, as they come from different countries like China, India or Ukraine.

The methodology followed in the classroom was to propose the students several open problematic situations related to physical and chemical changes and then to compare the written productions of students when solving these open problematic situations before and after using the MPSI methodology. With the first situation called Which paper absorbs more?, students solved an open problem without following a specific methodology. With the second problem situation, How do I separate a mixture of iron, copper, cork and salt?, the students used the MPSI for the first time. And with the third open problem situation, What makes bread more spongy?, they used the same methodology again. In this way we can see the difference between using or not the MPSI methodology by comparing the first two problems, and we can see the difference in using this methodology repeatedly by comparing the last two problems.

For qualitative and descriptive analysis of written solutions, we matched the MPSI phases with the dimensions of competence, and different response levels were pre-set to facilitate their quantification. In addition, achievement indicators have been determined to give an overview of what happened. Likewise, to discuss developments in each competence dimension, Bliss-type diagrams were made. We have also analysed a satisfaction questionnaire completed by students in order to know their acceptance of the methodology. 
The results showed that:

1. The introduction of MPSI allows a significant improvement of students in aspects of scientific competence related to the design of problem solving and its implementation. This also introduces an enhancement of other aspects such as hypotheses emission or analysis of the problem situation and its results.

2. The use of MPSI within the learning sequence Physical changes and chemical changes has helped diversification students to achieve higher competence levels, mainly in areas such as hypothesis emission, results analysis or experiment development.

3. $46 \%$ of students have a positive valuation of $M P S I$ versus $21 \%$ of students with a negative valuation, the latter mainly due to the higher level of effort involved in this working method.

In conclusion, we can say that the Methodology of Problem-Solving as an Investigation (MPSI) produces good results in the development of different aspects of scientific competence in students from a diversification program, and it does so with appreciable satisfaction from them. 